\title{
12 Das Wichtigste für den Arzt kurzgefasst
}

Informieren Sie sich nach Möglichkeit vor der Behandlung von Patienten, die nicht mehr einwilligungsfähig sind, ob

\) eine Vollmacht erteilt worden ist, die auch die Heilbehandlung umfasst,

\) ein gesetzlicher Betreuer mit dem Aufgabenkreis „Heilbehandlung" bestellt ist,

\) eine Patientenverfügung erstellt wurde.

Ist eine Vollmacht erteilt worden oder ein gesetzlicher Betreuer bestellt, holen Sie von dieser Person stellvertretend für den Patienten die Einwilligung für Ihre ärztliche Behandlung ein.

Im Notfall, wenn keine Zeit bleibt, die Einwilligung eines Bevollmächtigten oder gesetzlichen Betreuers einzuholen, handeln Sie lege artis im objektiven Interesse Ihres Patienten.

Ist keine Vollmacht erteilt und noch kein Betreuer bestellt, regen Sie beim Betreuungsgericht eine Betreuerbestellung an.

Die Umsetzung einer Patientenverfügung bzw. des mutmaßlicher Willens erfolgt im Einvernehmen mit dem Bevollmächtigten/Betreuer. Nur im Dissensfall ist eine gerichtliche Genehmigung erforderlich.

Ist kein Bevollmächtigter oder Betreuer vorhanden, können Sie eine Patientenverfügung ausnahmsweise auch ohne Beteiligung eines Stellvertreters direkt befolgen, wenn Sie keinerlei Zweifel an ihrer Validität haben. Andernfalls regen Sie die Bestellung eines Betreuers an, mit dem zusammen Sie die Patientenverfügung interpretieren und klären, ob der Wille des Patienten bezüglich der aktuellen Behandlungssituation festgestellt werden kann.

Kann weder ein schriftlicher Wille noch ein mutmaßlicher Wille festgestellt werden, gilt in dubio pro vita, solange lebensverlängernde Maßnahmen indiziert sind.

Falls Ihnen die Entscheidung eines Bevollmächtigten oder eines gesetzlichen Betreuers bezüglich einer ärztlichen Maßnahme - oder deren Unterlassung - in keiner Weise vertretbar erscheint, rufen Sie das nächstgelegene Betreuungsgericht an und verlangen Sie eine gerichtliche Eilentscheidung. 